\title{
MPPT Based on Sinusoidal Extremum-Seeking Control in PV Generation
}

\author{
R. Leyva, ${ }^{1}$ C. Olalla, ${ }^{1}$ H. Zazo, ${ }^{1}$ C. Cabal, ${ }^{2}$ A. Cid-Pastor, ${ }^{1}$ I. Queinnec, ${ }^{2,3}$ and C. Alonso ${ }^{2,3}$ \\ ${ }^{1}$ DEEEA, Universitat Rovira i Virgili, Avinguda Paisos Catalans 26, 43007 Tarragona, Spain \\ ${ }^{2}$ LAAS, CNRS, 7 Avenue du Colonel Roche, 31077 Toulouse, France \\ ${ }^{3}$ Université de Toulouse, UPS, INSA, INP, ISAE, LAAS, 31077 Toulouse, France \\ Correspondence should be addressed to R. Leyva, ramon.leyva@urv.cat
}

Received 25 October 2011; Accepted 8 December 2011

Academic Editor: Jean-Louis Scartezzini

Copyright (c) 2012 R. Leyva et al. This is an open access article distributed under the Creative Commons Attribution License, which permits unrestricted use, distribution, and reproduction in any medium, provided the original work is properly cited.

The paper analyses extremum-seeking control technique for maximum power point tracking circuits in PV systems. Specifically, the paper describes and analyses the sinusoidal extremum-seeking control considering stability issues by means a Lyapunov function. Based on this technique, a new architecture of MPPT for PV generation is proposed. In order to assess the proposed solution, the paper provides some experimental measurements in a $100 \mathrm{~W}$ prototype which corroborate the effectiveness of the approach.

\section{Introduction}

Photovoltaic panels require adapting the output-port voltage to extract the maximum deliverable power when weather conditions change. Such energy adaptation should have a good performance; that is, the operation point should be ensured to be near to the maximum and the adapting electronic system should behave reliably and efficiently. A reliable behavior means that the adaptation mechanism is dynamically stable and does not induce great stress to its components. Efficiency of the adapting electronic system depends on the losses in switching converters which adapt the voltage levels between the PV panels and the loads [1].

There exist several approaches to implement maximum power point tracking (MPPT) in PV systems. Remarkable surveys on this subject can be found in $[2,3]$. In their survey paper, Onat [2] classifies MPPT algorithms in two categories: indirect and direct methods. The indirect methods use prior information or a mathematical characterization of the PV panel and take measurements far from the desired operating point in order to estimate the voltage or current at maximum power point (MPP). The main drawback of this approach is that the PV system does not operate near to the MPP during the measuring time interval. Some instances of indirect methods are curve-fitting, look-up table, PV panel short-circuit, and PV panel open-circuit method. Another drawback of indirect methods is that they may cause large stress to components since measuring process may involve large changes in working conditions. The last two disadvantages have prompted some authors to use direct methods.

Direct methods take measurements of PV panel current and voltage and their corresponding time derivatives at the operating point in order to drive PV systems toward the maximum. Given that direct methods do not carry out abrupt changes in the operating point to make measurements, measuring frequency can be higher, and therefore these MPPT methods can faster track the optimum power point.

The most common direct MPPT methods are the P\&O algorithm [4] and the Incremental Conductance method [5]. Other techniques as fuzzy control $[6,7]$ and neural networks are also being used to develop MPPT controllers. This is because of the nonlinear relationships involved in photovoltaic systems. Tuning MPPT circuits are a difficult task, and a bad tuned MPPT circuit becomes instable when large disturbances occur. It is important to point out that instabilities may occur in any MPPT circuit when 
inherent delays in digital implementation or filters in analog implementations are not taken into account in the MPPT circuit design.

In parallel, with the development of MPPT methods in renewable energy field, some researchers have studied the technique named extremum seeking control (ESC) in the field of automatic control. This technique is concerned with algorithms that seek the maximum or the minimum of a nonlinear map. A system governed by ESC autooscillates around the optimum or the oscillation is forced by a sinusoidal signal. Autooscillating ESC algorithm is reported by Morosanov [8] and has been adapted to PV systems in [9]. Sinusoidal ESC has also been successfully applied to track the MPP of PV systems. This technique uses a sinusoidal perturbation to estimate the gradient of the voltage-power curve. Using this gradient value, ESC drives the PV system to the MPP.

Nevertheless, sinusoidal ESC is still in an incipient stage in PV systems, despite the research of Brunton et al. [10] where the ripple of the switching converter is used to estimate the gradient. It is also worth to mention the complementary approaches cited in [11]. Moreover, a supervisory strategy that uses sinusoidal ESC for cascaded $\mathrm{PV}$ architectures is reported in [12]. It is worth to note that MPPT implementations in [10-12] use digital devices.

Stability issues about sinusoidal ESC can be found in [13, 14]. Nevertheless, the nonlinear map of previous references is approximated by a parabola. Given that a parabolic approximation is not a good approximation of the powervoltage curve of a PV system, the stability analysis is only valid for small disturbances.

In the paper, we review the sinusoidal ESC technique. Also, we present a new stability demonstration based on Lyapunov analysis where we only assume that the nonlinear map is concave. This approach ensures the stability and therefore the reliability for all the range of operation of the $\mathrm{PV}$ system. The paper also presents a $100 \mathrm{~W}$ prototype which allows us to evaluate the effectiveness of the approach. The prototype differs from that of [10], since we use low cost analog devices. We report in detail experimental measurements that corroborate the effectiveness of the approach.

The paper is organized as follows. In Section 2, we review extremum-seeking control and specifically we analyze sinusoidal ESC taking into account stability aspects by means of a Lyapunov function. The global stability property ensures a correct behavior in front of abrupt changes in operating conditions. Section 3 describes a novel architecture for PV generation in which the MPPT circuit is based on sinusoidal ESC. In Section 4, we describe an experimental verification which allows us to assess the proposed MPPT solution. And, finally, we summarize the main ideas in Section 5.

\section{Sinusoidal Extremum-Seeking Control}

The objective of ESC is to force the operating point to be as close as possible to the optimum for a system described by an unknown nonlinear map with an only extremum (i.e., a maximum or a minimum).
Sinusoidal ESC principle, shown in Figure 1, can be summarized as follows: given a nonlinear input-output map, if a sinusoidal signal of little amplitude is added to the input signal $x$, the output signal $y$ oscillates around its average value. Both sinusoidal signals will be in phase if the input signal $x$ is smaller than the maximum of the nonlinear map and in counterphase if the input signal $x$ is larger than the maximum, as it is shown in Figure 1. It can be observed that when the input signal $x$ reaches maximum, then output signal $y$ doubles its frequency; also it can be appreciated that the amplitude of the ripple of the output signal $y$ depends on the slope of the curve $y=f(x)$. Also it can be noted that, when the signal $y$ is multiplied by a sinusoidal of the same frequency and phase, the multiplier output $g$ is positive before the maximum and negative at the right side of the maximum.

The method can be implemented by the schema of Figure 2. The schema consists of the nonlinear input-output map, an integrator and a detection block, and a small sinusoidal signal that is added to the map input. The detection block function demodulates the output signal $y$.

In the following sections, we describe the detection block function and analyze the stability condition of the schema shown in Figure 2.

2.1. Detection Block. Detection block output $u$ is proportional to the gradient of the nonlinear map as it is shown in the following paragraphs.

Given a signal $x$ at the output of the integrator block, the output of the nonlinear mapping $y$ will correspond to

$$
y=f\left(x+x_{0} \sin \left(\omega_{0} t\right)\right) .
$$

Considering that the sinusoidal perturbation is small, namely, given the condition $x_{0} \ll x$, then expression (1) can be approximated by its Taylor development as

$$
y \simeq f(x)+\frac{d f(x)}{d x} x_{0} \sin \left(\omega_{0} t\right) .
$$

Thus, using the trigonometric identity $2 \sin ^{2}\left(\omega_{0} t\right)=1-$ $\cos \left(2 \omega_{0} t\right)$, the signal at the output of the multiplier block $g$ can be approximated by

$$
\begin{aligned}
g \simeq & f(x) k x_{0} \sin \left(\omega_{0} t\right)+\frac{d f(x)}{d x} k x_{0}^{2} \sin ^{2}\left(\omega_{0} t\right) \\
= & \frac{1}{2} \frac{d f(x)}{d x} k x_{0}^{2}+f(x) k x_{0} \sin \left(\omega_{0} t\right) \\
& -\frac{1}{2} \frac{d f(x)}{d x} k x_{0}^{2} \cos \left(2 \omega_{0} t\right) .
\end{aligned}
$$

Now, considering that the low-pass filter attenuates completely the first and second harmonics, the expression of the filter output $u$ can be written as

$$
u=\frac{1}{2} \frac{d f(x)}{d x} k x_{0}^{2} * L^{-1}\left\{\frac{a}{s+a}\right\},
$$

where $*$ is the convolution operator, $L^{-1}$ stands for the inverse Laplace transform, and $L^{-1}\{a /(s+a)\}$ represents the filter impulsional response. 


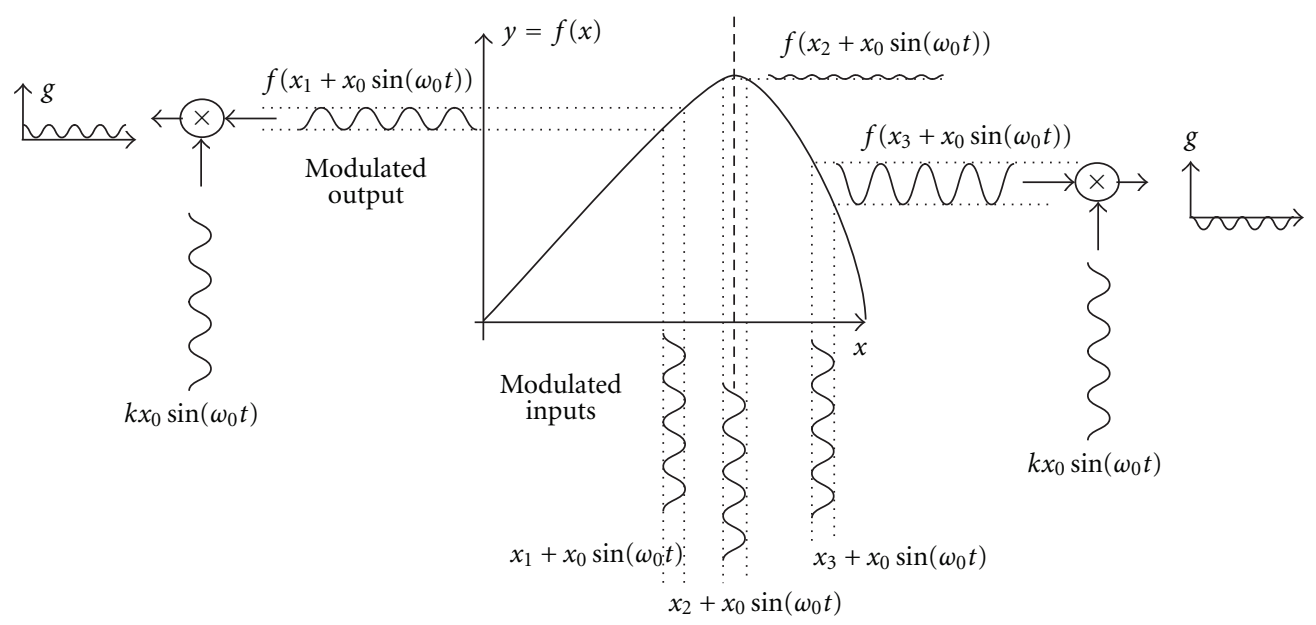

FIGURE 1: Sinusoidal ESC principle.

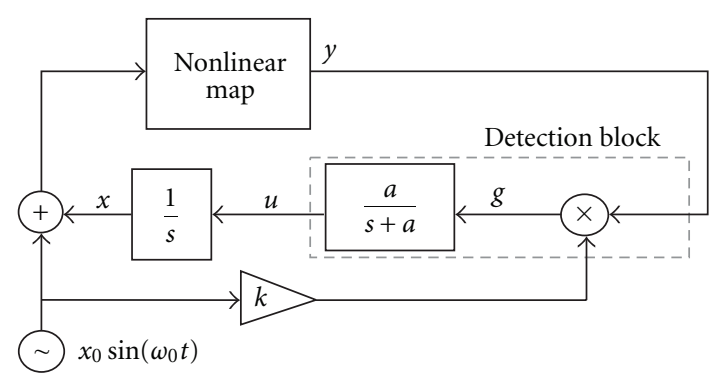

Figure 2: Sinusoidal ESC schema.

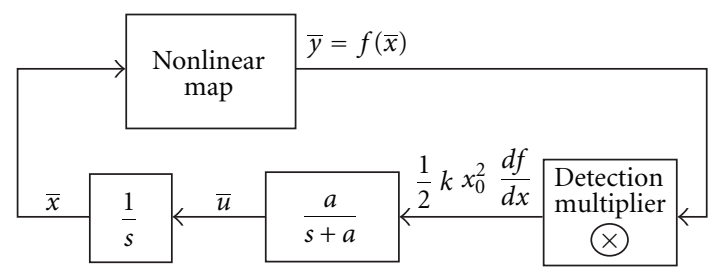

FIgURE 3: Averaged-signal extremum-seeking block diagram.

Therefore, under the assumptions of small amplitude of the sinusoidal perturbation and enough attenuation of the harmonics of multiplier output, it can be stated that the output signal of the detection block $u$ is proportional to the derivative of the nonlinear map output with respect to its input, namely, the gradient $d f(x) / d x$.

2.2. Dynamical Stability Analysis. The following analysis is carried out by means of averaged signals. Averaged signals and real signals differ only in a magnitude which depends on $x_{0}$, and we have assumed that the sinusoidal perturbation $x_{0}$ is small.

Averaged signals that take part in the sinusoidal extremum-seeking circuit are $\bar{x}=(1 / T) \int_{0}^{T}\left(x+x_{0} \sin \left(\omega_{0} t\right)\right) d t, \bar{y}=$ $(1 / T) \int_{0}^{T} y(t) d t$, and $\bar{u}=(1 / T) \int_{0}^{T} u(t) d t$, being $T=2 \pi / \omega_{0}$. The averaged output of the nonlinear map is $\bar{y}=\overline{f(x)}$, and, from expression (2), we can state that $\overline{f(x)} \simeq f(\bar{x})$, and, thus, dynamical relations of averaged signals can be represented by Figure 3.

Therefore, denoting the constants terms as $k_{1}$, that is, $k_{1}=k x_{0}^{2} / 2$, the averaged dynamical behavior can be described as

$$
\begin{gathered}
\dot{\bar{x}}=\bar{u}, \\
\dot{\bar{u}}=-a \bar{u}+a k_{1} \frac{d f}{d x},
\end{gathered}
$$

where the first row indicates the integrator differential equation and the second row the filter differential equation.

We assume that $f$ has an only maximum and is a concave function; thus the second derivative or Hessian is negative, that is, $d^{2} f / d x^{2}<0$.

It can be observed that the time derivative of the gradient corresponds to

$$
\frac{d}{d t}\left(\frac{d f}{d x}\right)=\frac{d^{2} f}{d x^{2}} \dot{\bar{x}}=\frac{d^{2} f}{d x^{2}} \bar{u}
$$

Now, renaming the state variables as $z_{1}=d f / d x$ and $z_{2}=$ $\bar{u}$, we can express the averaged dynamical system as

$$
\begin{gathered}
\dot{z}_{1}=h\left(z_{1}\right) z_{2}, \\
\dot{z}_{2}=-a z_{2}+a k_{1} z_{1},
\end{gathered}
$$

being $h\left(z_{1}\right)=d^{2} f / d x^{2}<0$.

Then, we consider the following candidate Lyapunov function to prove the stability of the nonlinear system (7),

$$
V\left(z_{1}, z_{2}\right)=\frac{1}{2} z_{2}^{2}+\int_{0}^{z_{1}}\left(-a k_{1} \frac{1}{h\left(\alpha_{1}\right)} \alpha_{1}\right) d \alpha_{1} .
$$

First, in order to verify the positive definiteness of $V(z)$, we note that the existence of a continuous function $m\left(z_{1}\right)$ such that $m\left(z_{1}\right) \cdot z_{1}>0$, for all $z_{1} \neq 0$, implies that 


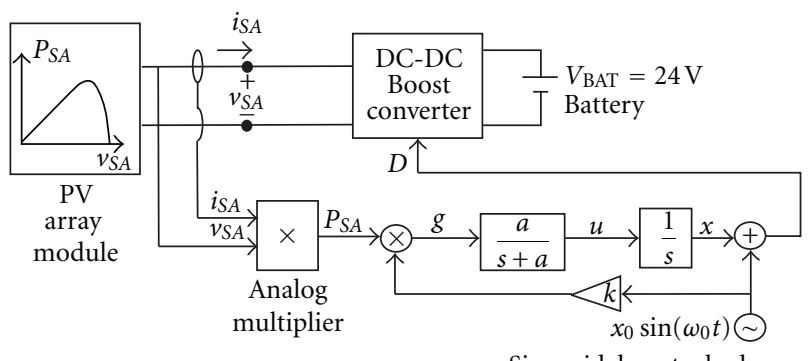

Sinusoidal-perturbed

Extremum-seeking controller

FIgURE 4: Proposed MPPT PV generator schema.

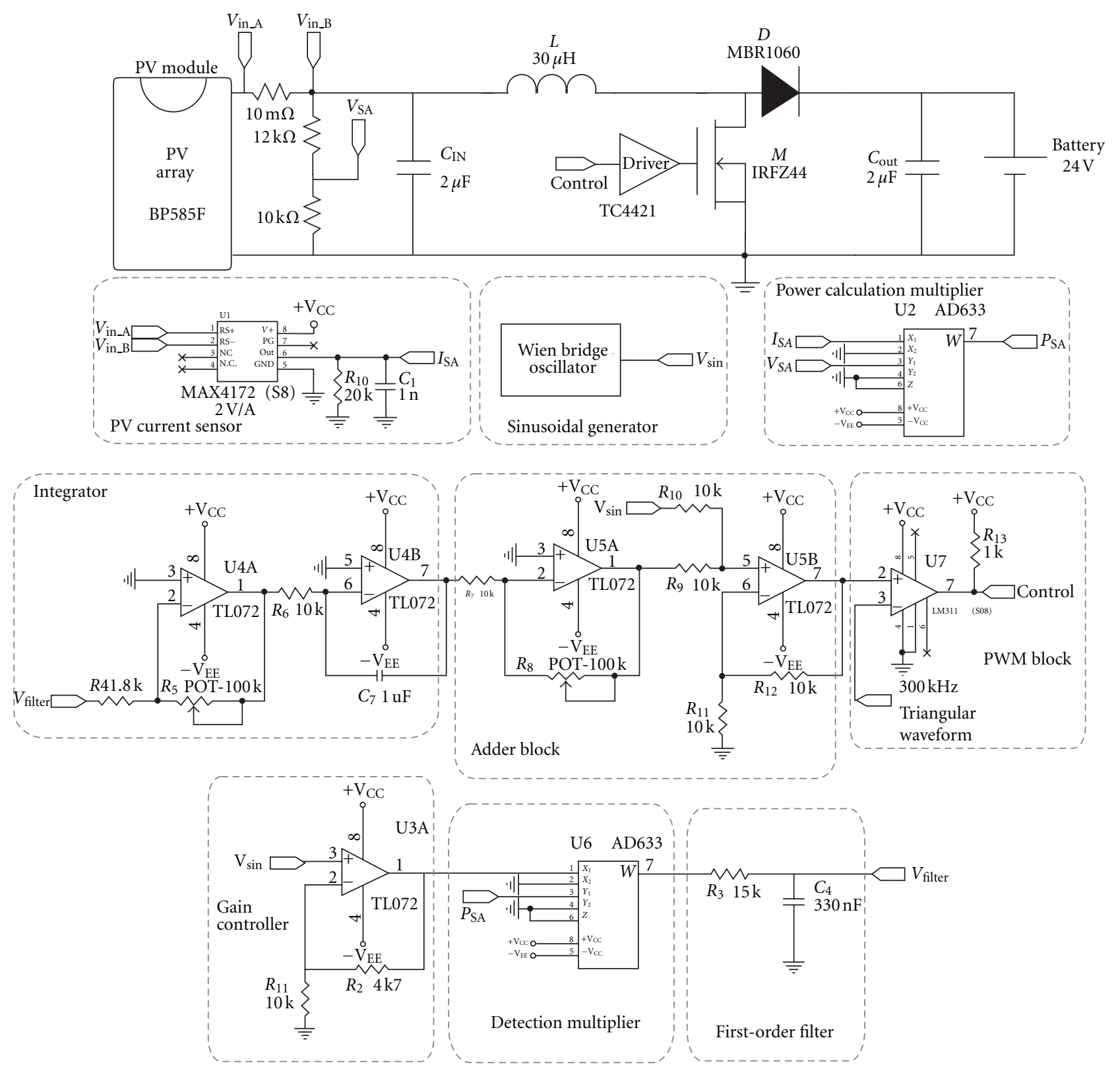

FIGURE 5: Prototype of PV generator with MPPT based on sinusoidal ESC. 
$\int_{0}^{z_{1}} m\left(\alpha_{1}\right) d \alpha_{1}>0$. It can be corroborated given that it is true for positive and negative $z_{1}$, that is,

$$
\begin{gathered}
z_{1}>0 \Longrightarrow m\left(\alpha_{1}\right)>0, \\
\alpha_{1} \in\left(0, z_{1}\right) \Longrightarrow \int_{0}^{z_{1}} m\left(\alpha_{1}\right) d \alpha_{1}>0, \\
z_{1}<0 \Longrightarrow m\left(\alpha_{1}\right)<0, \\
\alpha_{1} \in\left(z_{1}, 0\right) \Longrightarrow \int_{0}^{z_{1}} m\left(\alpha_{1}\right) d \alpha_{1}=-\int_{z_{1}}^{0} m\left(\alpha_{1}\right) d \alpha_{1}>0 .
\end{gathered}
$$

Now taking the function $m\left(z_{1}\right)$ as $m\left(z_{1}\right)=$ $-a k_{1}\left(1 / h\left(z_{1}\right)\right) z_{1}$, it can be seen that, since $a>0, k_{1}>0$, and $h\left(z_{1}\right)<0$, then $m\left(z_{1}\right) \cdot z_{1}>0$, for all $z_{1} \neq 0$. Therefore, $\int_{0}^{z_{1}}\left(-a k_{1}\left(1 / h\left(\alpha_{1}\right)\right) \alpha_{1}\right) d \alpha_{1}>0$, and, thus, $V\left(z_{1}, z_{2}\right)$ is positive definite.

In addition the time derivative of $V\left(z_{1}, z_{2}\right)$ corresponds to

$$
\dot{V}\left(z_{1}, z_{2}\right)=z_{2} \dot{z}_{2}+\left(-a k_{1} \frac{1}{h\left(z_{1}\right)} z_{1}\right) \dot{z}_{1}
$$

And substituting the state variables derivatives according to (7) in the time derivative of the Lyapunov function, (10) can be rewritten as

$$
\dot{V}\left(z_{1}, z_{2}\right)=-a z_{2}^{2}
$$

Hence, given that $V\left(z_{1}, z_{2}\right)$ is positive definite and $\dot{V}\left(z_{1}, z_{2}\right)$ is seminegative definite, the stability of the system is proven. It is also straight to establish the asymptotical stability of the system (7), by using the Lasalle invariance principle [15] since the only invariant of system (7) for which $\dot{V}=0$ is the origin.

We should remark that the only assumption is that the nonlinear map has an only máximum, that is, $d^{2} f / d x^{2}<$ 0 . The nonlinear map corresponding to the power-voltage curve of a PV panel has an only maximum. In the following section, we adapt the analyzed algorithm as MPPT circuit for a PV system.

Moreover, we remark this prove ensures the stability and therefore the reliability for all the range of operation of the PV system. An analysis that only considers the stability in front of small signal disturbances can be found in [14].

\section{Electronic Architecture of the Sinusoidal ESC as MPPT Circuit}

The solar panel model, as that depicted in Figure 4, can be represented by the function $P_{\mathrm{SA}}=f_{1}\left(v_{\mathrm{SA}}\right)$ that relates the output power $P_{\mathrm{SA}}$ of a solar array with respect its port voltage $v_{\mathrm{SA}}$. The function is a concave with uniform irradiation, namely, $d^{2} f_{1}\left(v_{\mathrm{SA}}\right) / d v_{\mathrm{SA}}^{2}<0$. It means that the function $P_{\mathrm{SA}}=$ $f_{1}\left(v_{\mathrm{SA}}\right)$ has a unique maximum at MPP.

We adapt the PV panel output voltage to the load (in our case a battery) by means of a boost DC-DC switching converter, as shown in Figure 4. In addition, the relation $v_{\mathrm{SA}}=f_{2}(D)$ between the duty-cycle $D$ and the input voltage $v_{\mathrm{SA}}$ in the boost converter corresponds to $v_{\mathrm{SA}}=(1-D) V_{g}$

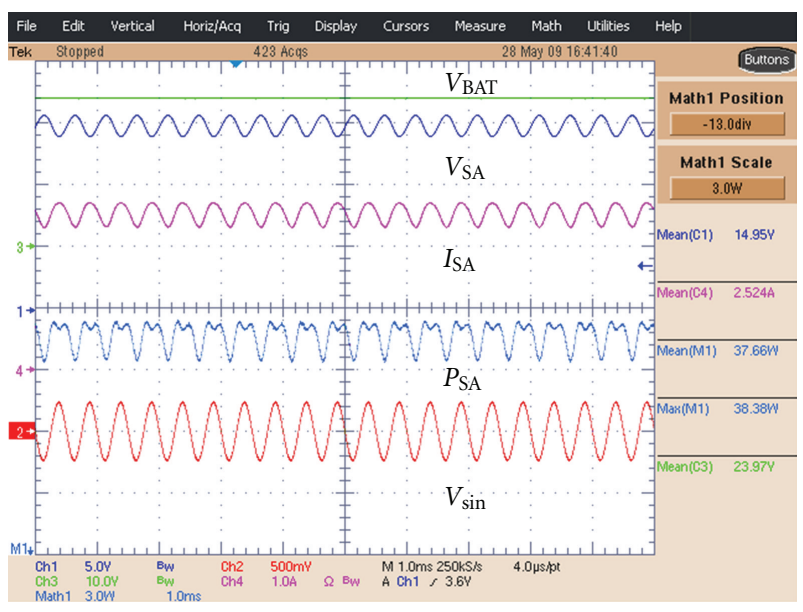

FIGURE 6: Steady-state waveforms.

for $D \in[0,1]$ in a time scale much slower than its switching frequency and its time constants. Then, it can be stated that $v_{\mathrm{SA}}=f_{2}(D)$ is a monotonically increasing function inside the duty-cycle interval, and the composite function $P_{\mathrm{SA}}(D)=$ $f_{1}\left(f_{2}(D)\right)=f_{1} \circ f_{2}(D)$ is a concave function [16], namely, $d^{2} f(D) / d D^{2}<0$. Therefore, signals in the schema of Figure 4 tend to the equilibrium, and the equilibrium corresponds to the maximum output power point, as stated in previous section.

\section{Experimental Verification}

In this section, we describe an electronic implementation of the schema depicted in Figure 4. We have built a $100 \mathrm{~W}$ prototype to verify the proposed approach. The prototype features are first described; then, we depict the obtained waveforms.

4.1. Prototype Description. Figure 5 illustrates the experimental prototype based on sinusoidal ESC. The DC-DC boost-like converter processes the power from an $85 \mathrm{Wp}$ monocrystalline photovoltaic array, whose nominal opencircuit voltage $v_{\mathrm{OC}}$ is $22.1 \mathrm{~V}$ and the nominal voltage value at the maximum power point is $18 \mathrm{~V}$. The power is stored in an acid-lead battery whose nominal voltage $V_{\text {BAT }}$ is $24 \mathrm{~V}$. The capacitor values are $2 \mu \mathrm{F}$, and inductor value is $30 \mu \mathrm{H}$ in the dc-dc converter, as Figure 5 depicts. The converter switching frequency is $300 \mathrm{KHz}$. Power measures use an IC AD633 analog multiplier.

Besides the PV module, the dc-dc converter and its PWM circuit, the battery, and the power calculating multiplier, there exists also an adder block which sums the sinusoidal dithering before the PWM block. The key parameters of the sinusoidal dithering generator are its amplitude and frequency. The generator amplitude must be much larger than the switching ripple but small enough since MPPT efficiency depends on it. In the prototype, the generator amplitude and frequency are, respectively, $0.5 \mathrm{Vpp}$ and $2 \mathrm{kHz}$, whereas the PWM sawtooth amplitude is $7 \mathrm{Vpp}$. The 


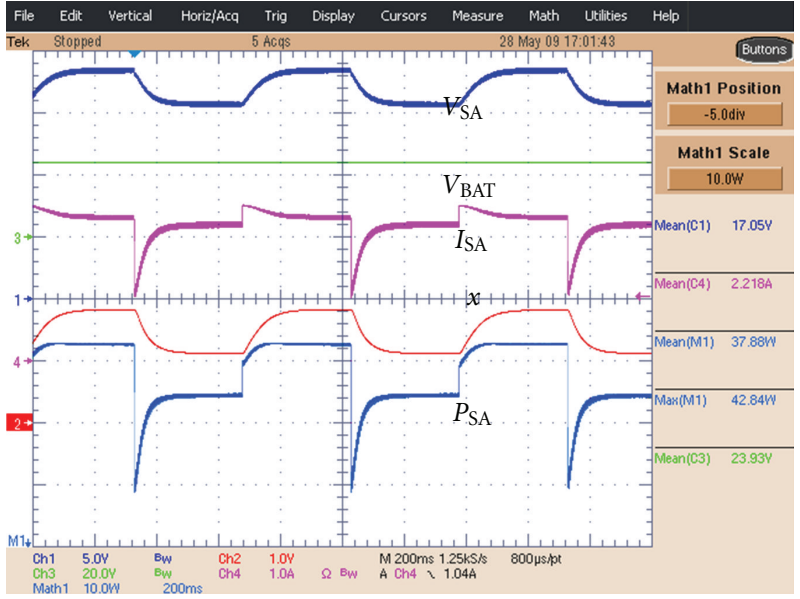

(a)

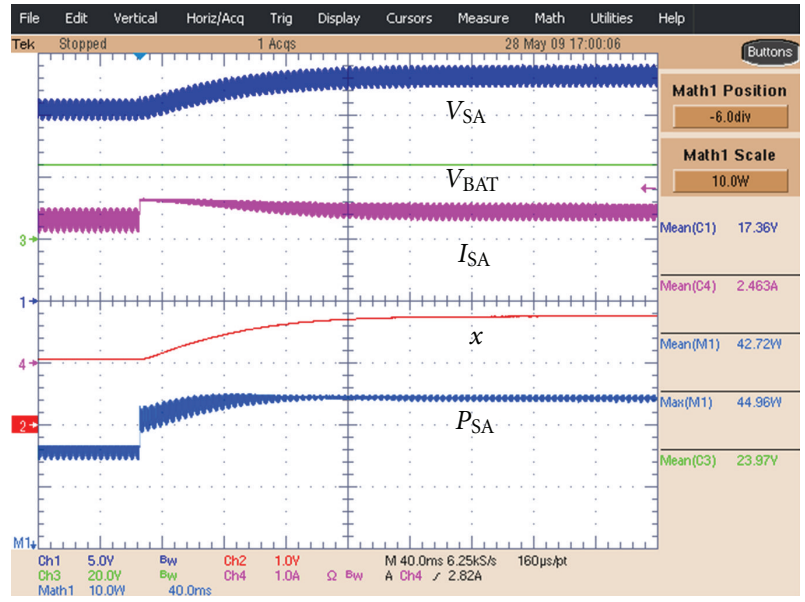

(b)

FIgURe 7: (a) Transient waveforms for voltage-pulsed signal in series with the PV panel. (b) Detail of transient for voltage pulse added to panel voltage.

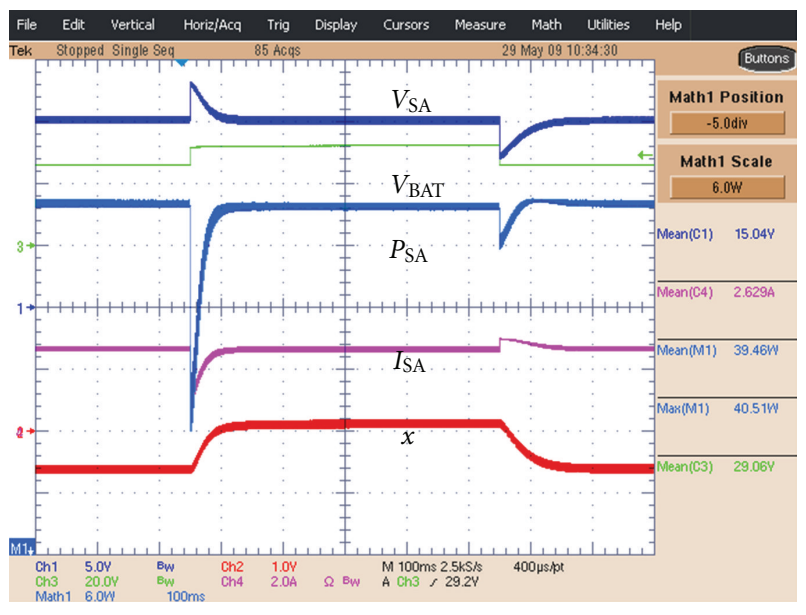

(a)

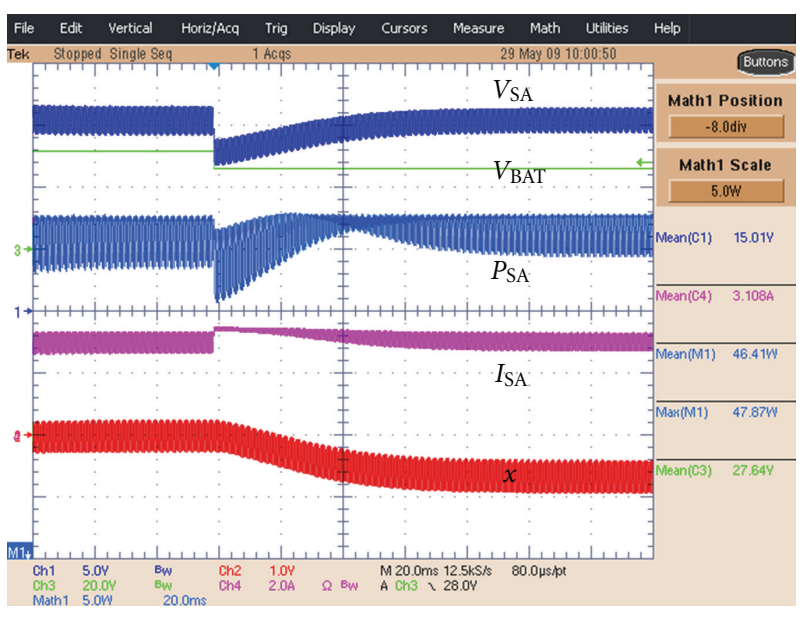

(b)

Figure 8: (a) Transient waveform when voltage pulse signal is added to battery voltage. (b) Detail of the transient waveforms during a decrease step of the battery voltage.

demodulating or detecting multiplication is also carried out by the IC AD633. A first-order filter is located after the detecting multiplier whose cut-off frequency is $200 \mathrm{~Hz}$ aprox. A tradeoff appears in the election of the crossover frequency since a higher value allows a faster behavior; nevertheless a lower value filters undesired harmonics better. The integrator block values must ensure that no saturation appears, and the controller gain must ensure stability and a suitable degree of damping. Their values are shown in Figure 5.

4.2. Measured Signals. In this subsection, we explain the behavior of the prototype in steady state, also when a $3 \mathrm{~V}$ pulse is put in series with photovoltaic panel, and when a $6 \mathrm{~V}$ pulse is put in series with the battery. Figure 6 shows the steady-state waveforms. Given maximum power and the mean power displayed in the figure, it can be derived that the MPPT efficiency is $\eta_{\text {MPPT }}=$ Mean Power/ Max Power $=$ $37.66 \mathrm{~W} / 38.38 \mathrm{~W}=98 \%$; a better efficiency can still be achieved, but we show cleaner waveforms with this efficiency level. It can also be noted that, during a half period of the voltage signal $V_{\mathrm{SA}}$, the power signal $P_{\mathrm{SA}}$ passes through the maximum; therefore, the system is effectively around the optimal point. Battery voltage $V_{\mathrm{BAT}}$ is $23.9 \mathrm{~V}$, and mean values of voltage and current are $14.95 \mathrm{~V}$ and $2.52 \mathrm{~A}$, respectively.

Figure 7(a) depicts the behavior when a voltage pulse appears in series with the PV panel. Signals from top to down are the following: panel output voltage $V_{\mathrm{SA}}$, battery voltage $V_{\mathrm{BAT}}$, panel output current $I_{\mathrm{SA}}$, integrator output $x$, and power $P_{\mathrm{SA}}$. It can be appreciated that when the system loses the MPP due to a pulsed variation, the system recovers 
its steady state very fast. Figure 7(b) shows a detail of the transition when a voltage pulse is added to the panel voltage.

Figure 8(a) shows the transient in front of a pulse variation of the battery voltage. The upper signal in the oscilloscope picture is the panel output voltage $V_{\mathrm{SA}}$ which recovers a value of $15 \mathrm{~V}$ after the transient. The second signal depicts the battery voltage $V_{\mathrm{BAT}}$ when a pulse variation of $6 \mathrm{~V}$ appears. The third signal corresponds to the power signal $P_{\mathrm{SA}}$, and it recovers the MPP in less than $20 \mathrm{~ms}$. The fourth signal is the panel output current $I_{\mathrm{SA}}$, whereas the lower signal shows the integrator output $x$. As can be viewed, in Figure 8 (b), after a step decrease of the battery voltage, the sinusoidal oscillation of the power seems to fold near the MPP, changing in $180^{\circ}$ the phase of the main harmonic of the signal.

\section{Conclusions}

The paper reviews the control technique named sinusoidal ESC. A novel architecture for MPPT photovoltaic generation is proposed based on this technique. Such technique provides a high efficient method to track the maximum power point, since it results in a small oscillation around the MPP. This oscillation depends on the amplitude of the sinusoidal modulator signal and is proportional to the slope of the powervoltage curve of the PV panel. Therefore, given that the slope is small near optimum, then the oscillation is small near the maximum and the MPPT has a very good performance. The paper also analyzes the stability of sinusoidal ESC for PV systems by means a Lyapunov function. In addition, in order to verify the effectiveness of the approach, we describe the implementation a prototype for a $100 \mathrm{Wp}$ solar generator, which provides accurate measures which are in good agreement with previous derivation.

\section{References}

[1] S. Sladić, S. Skok, and D. Nedeljković, "Efficiency considerations and application limits of single-phase active power filter with converters for photoenergy applications," International Journal of Photoenergy, vol. 2011, Article ID 643912, 8 pages, 2011.

[2] N. Onat, "Recent developments in maximum power point tracking technologies for photovoltaic systems," International Journal of Photoenergy, vol. 2010, Article ID 245316, 11 pages, 2010.

[3] C. Cabal, Optimisation énergétique de l'étage d'adaptation électronique dédié à la conversion photovoltaïque, Thèse de Doctorat, Université de Toulouse, 2008, (In French).

[4] N. Femia, G. Petrone, G. Spagnuolo, and M. Vitelli, "Optimization of perturb and observe maximum power point tracking method," IEEE Transactions on Power Electronics, vol. 20, no. 4, pp. 963-973, 2005.

[5] K. H. Hussein, I. Muta, T. Hoshino, and M. Osakada, "Maximum photovoltaic power tracking: an algorithm for rapidly changing atmospheric conditions," IEE Proceedings: Generation, Transmission and Distribution, vol. 142, no. 1, pp. 59-64, 1995.

[6] Subiyanto, A. Mohamed, and H. Shareef, "Hopfield neural network optimized fuzzy logic controller for maximum power point tracking in a photovoltaic system," International Journal of Photoenergy, vol. 2012, Article ID 798361, 13 pages, 2012.

[7] M. Taherbaneh, A. H. Rezaie, H. Ghafoorifard, K. Rahimi, and M. B. Menhaj, "Maximizing output power of a solar panel via combination of sun tracking and maximum power point tracking by fuzzy controllers," International Journal of Photoenergy, vol. 2010, Article ID 312580, 13 pages, 2010.

[8] I. S. Morosanov, "Methods of extremum control," Automation and Remote Control, vol. 18, pp. 1077-1092, 1957.

[9] R. Leyva, C. Alonso, I. Queinnec, A. Cid-Pastor, D. Lagrange, and L. Martínez-Salamero, "MPPT of photovoltaic systems using extremum seeking control," IEEE Transactions on Aerospace and Electronic Systems, vol. 42, no. 1, pp. 249-258, 2006.

[10] S. L. Brunton, C. W. Rowley, S. R. Kulkarni, and C. Clarkson, "Maximum power point tracking for photovoltaic optimization using ripple-based extremum seeking control," IEEE Transactions on Power Electronics, vol. 25, no. 10, Article ID 5460938, pp. 2531-2540, 2010.

[11] A. M. Bazzi and P. T. Krein, "Concerning maximum power point tracking for photovoltaic optimization using ripplebased extremum seeking control," IEEE Transactions on Power Electronics, vol. 26, no. 6, Article ID 5959287, pp. 1611-1612, 2011.

[12] A. I. Bratcu, I. Munteanu, S. Bacha, D. Picault, and B. Raison, "Cascaded DC-DC converter photovoltaic systems: power optimization issues," IEEE Transactions on Industrial Electronics, vol. 58, no. 2, Article ID 5416284, pp. 403-411, 2011.

[13] K. B. Ariyur and M. Krstić, Real-Time Optimization by Extremum-Seeking Control Hoboken, Wiley-Interscience, Hoboken, NJ, USA, 2003.

[14] R. Leyva, P. Artillan, C. Cabal, B. Estibals, and C. Alonso, "Dynamic performance of maximum power point tracking circuits using sinusoidal extremum seeking control for photovoltaic generation," International Journal of Electronics, vol. 98, no. 4, pp. 529-542, 2011.

[15] H. K. Khalil, Nonlinear Systems, Prentice-Hall, Englewood Cliffs, NJ, USA, 2nd edition, 1996.

[16] S. Boyd and L. Vandenberghe, Convex Optimization, Cambridge University Press, Cambridge, UK, 2004. 


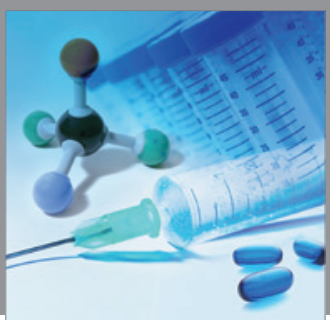

International Journal of

Medicinal Chemistry

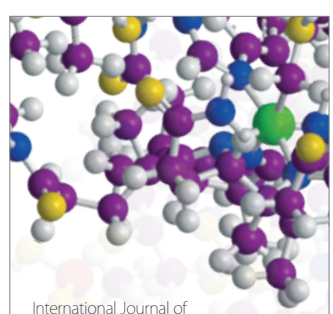

Carbohydrate Chemistry

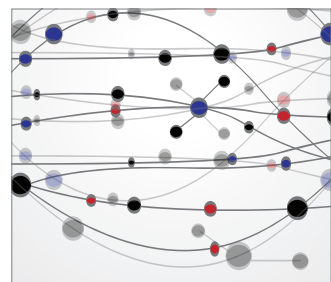

The Scientific World Journal
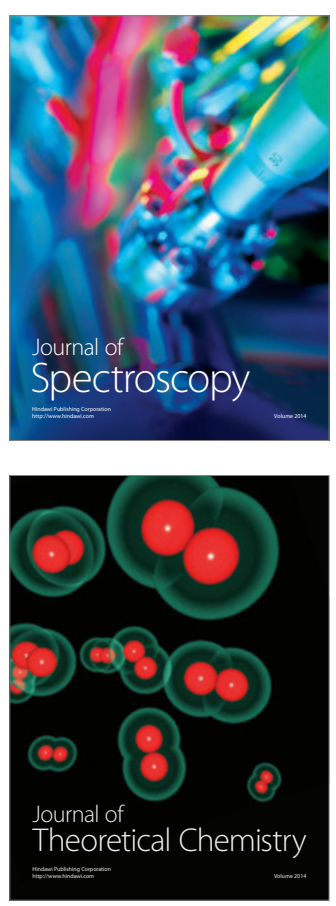
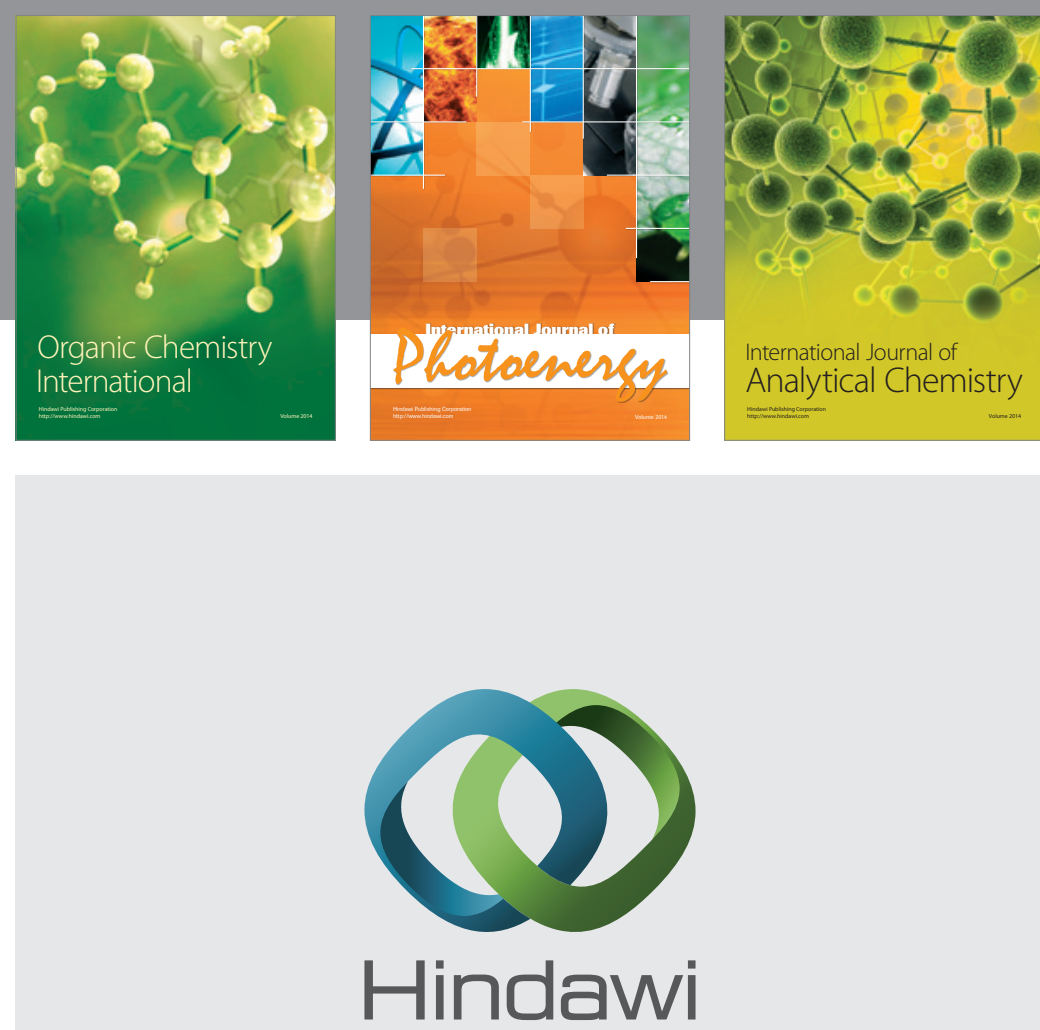

Submit your manuscripts at

http://www.hindawi.com
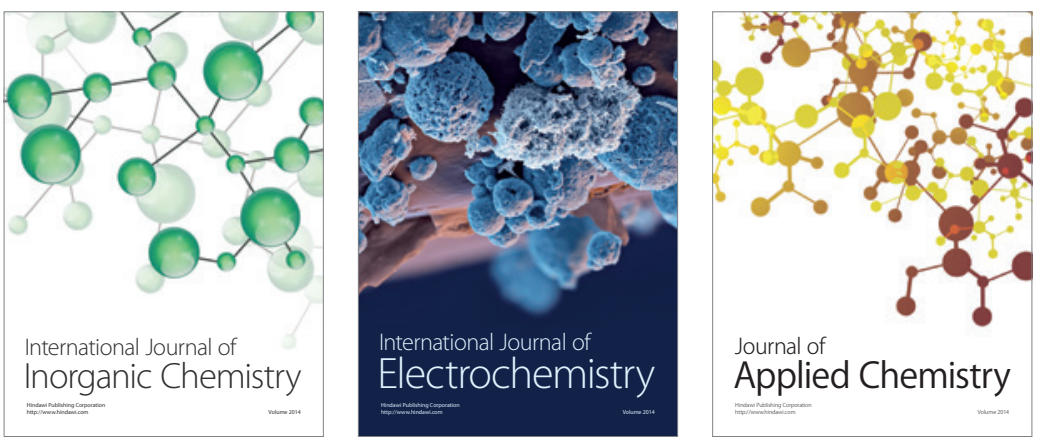

Journal of

Applied Chemistry
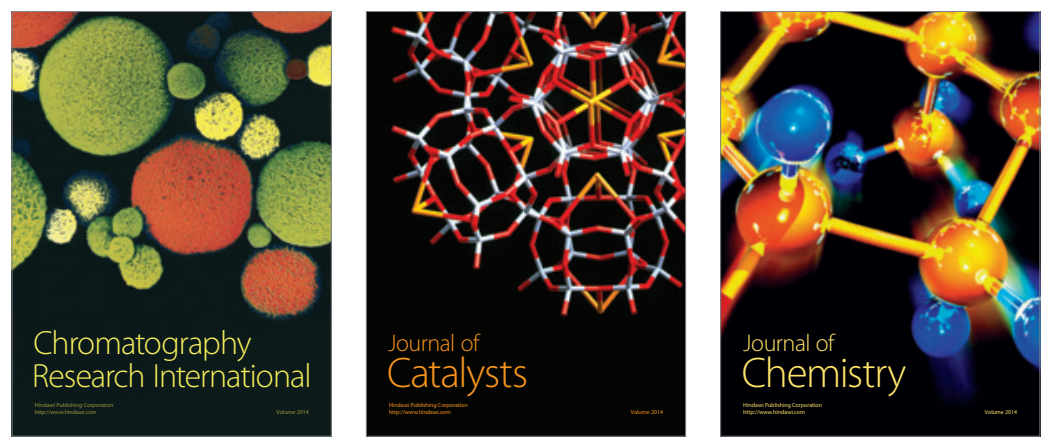
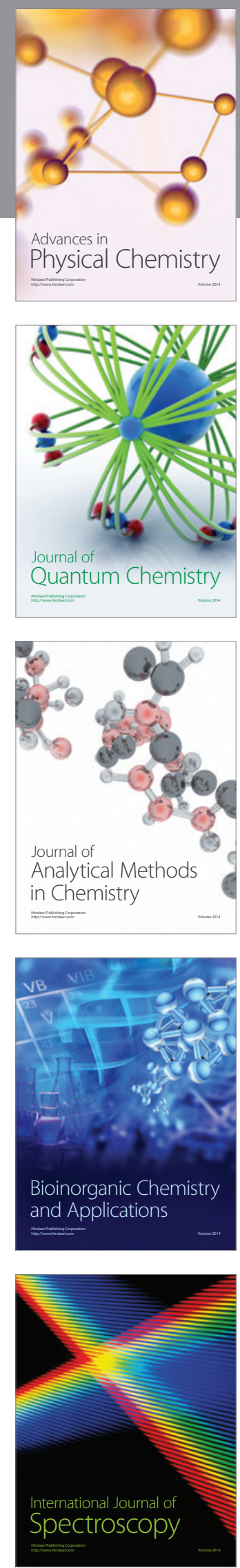